\title{
DETERMINACIÓN DE PRODUCCIÓN DE METANO EN CAPRINOS ALIMENTADOS CON DIETAS CON DISTINTOS CEREALES
}

\author{
DETERMINATION OF METHANE PRODUCTION IN GOATS FED \\ DIFFERENT CEREAL DIETS
}

\begin{abstract}
López, M.C. ${ }^{1 *}$, Ródenas, L. ${ }^{1}$, Piquer, O. ${ }^{2}$, Cerisuelo, A. ${ }^{3}$, Cervera, C. ${ }^{1}$ y Fernández, C. ${ }^{1}$
${ }^{1}$ Instituto de Ciencia y Tecnología Animal. Universidad Politécnica de Valencia. Valencia. España. *malolu@upvnet.upv.es

${ }^{2}$ Departamento de Producción Animal. Universidad Cardenal Herrera CEU. Moncada. Valencia. España. ${ }^{3}$ Centro de Investigación y Tecnología Animal (CITA-IVIA). Instituto Valenciano de Investigaciones Agrarias. Moncada. Valencia. España.
\end{abstract}

\section{PaLABRAS CLAVE ADICIONALES}

Ym.

\section{RESUMEN}

Se utilizaron tres cabras primíparas de raza Murciano-Granadina de un año y medio de edad y en estado de mantenimiento, con un peso medio de $33,08 \pm 2,1 \mathrm{~kg}$, con el objeto de determinar la producción de metano al administrarles dos dietas que difirieron únicamente en el tipo de cereal (grano de cebada o maíz). Se realizaron dos pruebas de digestibilidad consecutivas mediante la administración de las dos dietas. Tras un periodo de adaptación de 7 días se analizó la digestibilidad aparente durante 5 días. La producción de metano $\left(\mathrm{CH}_{4}\right)$ se determinó por calorimetría indirecta mediante una máscara de circuito abierto adaptada a pequeños rumiantes. $\mathrm{El} \mathrm{CH}_{4}$ se midió el día 4 de la prueba de digestibilidad, antes de administrar la ración diaria y se realizaron tres medidas más cada 35 minutos mientras comían. No se observaron diferencias significativas en el consumo de materia seca (con $758 \mathrm{~g} \mathrm{MS/cabra} \mathrm{y}$ día de media), ni entre los coeficientes de digestibilidad de materia seca de las dos dietas (CDMS= $69,2 \%$ de media). Durante la comida se observaron diferencias $(p<0,05)$ en la producción de metano con valores de $0,69 \mathrm{l} / \mathrm{h}$ para la dieta que contenía grano de cebada frente a $0,77 \mathrm{l} / \mathrm{h}$ para la dieta con grano de maíz. Cuando se expresó la producción de $\mathrm{CH}_{4}$ sobre la ingestión de energía (Ym) no se observaron diferencias significativas entre dietas, y el valor medio para las dietas mixtas estudiadas fue de 4,8 .

\section{AdDITIONAL KEYWORDS}

Ym.

\section{SUMMARY}

Three primiparous 1.5 year old Murciano-Granadina female goats in maintenance were used. Their average body weight was $33.08 \pm 2.1 \mathrm{~kg}$. The objective was to determine methane production in two diets differing of the type of cereal grain (barley or corn). Two consecutive digestibility trials were conducted using diets differing in the cereal type (barley or maize grains). The adaptation period has a duration of 7 days and the digestibility period 5 days. Methane production $\left(\mathrm{CH}_{4}\right)$ was determined by indirect calorimetry with an open circuit mask adapted to small ruminants. Methane production was determined on day four of digestibility trial, before feeding and three more measurements every 35 minutes during feeding. There were no significant differences neither in dry matter intake (758 g MS/goat and day on average) nor in dry matter digestibility coefficients (CDMS $=69.2 \%$ on average). Significant differences $(p<0.05)$ were observed in the methane production during the feeding time with levels of 0.69 and $0.77 \mathrm{l} / \mathrm{h}$ for the barley and maize based diets, respectively. No significant differences between diets were observed when $\mathrm{CH}_{4}$ was expressed on gross energy intake basis, and the value obtained for the experimental mixed diets was 4.8 .

\section{INTRODUCCIÓN}

En los últimos años se ha producido un 


\section{LÓPEZ, RÓDENAS, PIQUER, CERISUELO, CERVERA Y FERNÁNDEZ}

considerable debate y una creciente preocupación en torno al incremento de las emisiones de gases con efecto invernadero, como resultado de la actividad humana en general, y de la ganadería en particular. En cuanto al impacto de la ganadería, se reconoce que las actividades ganaderas emiten cantidades considerables de gases invernadero: dióxido de carbono $\left(\mathrm{CO}_{2}\right)$, metano $\left(\mathrm{CH}_{4}\right)$ y óxido nitroso $\left(\mathrm{N}_{2} \mathrm{O}\right)$, que contribuyen de manera importante al cambio climático.

La ganadería es responsable de cerca del $23 \%$ de las emisiones de $\mathrm{CH}_{4}$ globales de origen antropogénico (Khalil, 2000), debido mayoritariamente a que los rumiantes emiten metano durante la digestión del alimento en el rumen. En España la contribución de la ganadería a las emisiones de $\mathrm{CH}_{4}$ supera el $35 \%$, considerando solamente la fermentación entérica, si además se incluye la gestión del estiércol, la ganadería es responsable del orden de un $60 \%$ del total de las emisiones de $\mathrm{CH}_{4}$ (Ministerio de Medio Ambiente, 2007).

El metano es un importante gas de efecto invernadero, su concentración en la atmósfera ha aumentado rápidamente y se ha multiplicado por dos desde el comienzo de la Era Industrial (Moss et al., 2000; IPCC, 2001; Wuebbles y Hayhoe, 2002). Por otro lado, su tiempo de permanencia en la atmósfera es reducido, por tanto, se puede actuar a corto plazo disminuyendo su concentración.

Los animales durante la digestión producen metano de forma natural, principalmente debido a los microorganismos presentes en el aparato digestivo que fermentan el alimento consumido, produciéndose metano que se elimina principalmente por exhalación o eructo. La cantidad de metano producida depende de diversos factores: el tipo de animal (rumiante o monogástrico), estado fisiológico, nivel de alimentación y el tipo de dieta (forraje, dieta mixta o concentrada).

Entre los tipos de animal, los rumiantes emiten significativamente mayores cantidades de $\mathrm{CH}_{4}$ que los no-rumiantes debido a la elevada población de microorganismos productores de metano que habitan en el retículo-rumen (Crutzen et al., 1986; Moss et al., 2000). El metano, por lo tanto, es un producto de desecho nutricional (O'Kelly y Spiers, 1992; Lana et al., 1998), y puede representar entre el 2 y $11 \%$ de la energía bruta consumida por el rumiante (Johnson y Johnson, 1995).

El tipo de alimento es el otro factor determinante de las cantidades de metano producido por el rumiante, ya que es el substrato donde actuarán los microorganismos productores de metano. En el caso de los forrajes, se ha sugerido el uso de fuentes de fibra lignificadas como forma de reducir las emisiones de metano (Kreuzer y Hindrichsen, 2006). Un aumento en la ración de la proporción de concentrado en relación al forraje supone también un descenso considerable de la concentración de $\mathrm{H}$, de microorganismos metanogénicos y de la producción de $\mathrm{CH}_{4}$ (Van Soest, 1994; Lana et al., 1998). Y además, el tipo de concentrado incluido en la dieta (diferente proporción de endospermo harinoso o córneo) va a suponer modificaciones en la producción de metano como sugieren Beauchemin y McGinn(2005).

El objetivo del presente trabajo consistió en determinar la producción de metano en cabras Murciano-Granadinas en mantenimiento, alimentadas con dietas que difieren en el tipo de cereal: concretamente dietas conteniendo cebada grano o maíz grano.

\section{MATERIAL YMÉTODOS}

\section{AnImales ydietas}

Se utilizaron 3 cabras hembras de raza Murciano-Granadina de un año y medio de edad y en estado de mantenimiento. Dichas cabras pertenecen a la Granja Experimental del Departamento de Ciencia Animal en la Universidad Politécnica de Valencia. Previo 


\section{DETERMINACIÓN DE PRODUCCIÓN DE METANO EN GANADO CAPRINO}

al experimento se seleccionaron los animales con la misma edad y se pesaron en una báscula para pequeños rumiantes (Grupanor Cercampo S.A. Madrid, España) con el objeto de seleccionar 3 animales homogéneos en cuanto a su peso vivo $(33,08 \pm 2,1 \mathrm{~kg})$ y edad (1,5 años). Una vez seleccionadas las cabras se subieron a las jaulas metabólicas donde se alojaron durante el periodo de adaptación (7 días) y la posterior fase experimental ( 5 días).

Las dos dietas experimentales que recibieron las cabras se formularon siguiendo las recomendaciones de Lachica y Aguilera (2003) e INRA (2007). Como software se utilizó el programa CAPRA v1 (2008) y las materias primas que se utilizaron fueron heno de alfalfa, cebada, maíz, harina de girasol y bloque vitamínico-mineral. Las dietas se diferenciaron principalmente en

Tabla I. Ingredientes y composición química de las dietas experimentales. (Ingredients and chemical composition of experimental diets).

\begin{tabular}{lcc}
\hline & \multicolumn{2}{c}{ Dietas } \\
Ingredientes, g/kg & Cebada & Maíz \\
\hline Heno alfalfa & 718 & 751 \\
Cebada & 179 & - \\
Maíz & - & 141 \\
Harina girasol, pellet & 90 & 94 \\
Bloque vit-mineral ${ }^{1}$ & 13 & 14 \\
\hline Composición química, \% MS & Cebada & Maíz \\
MS (\%) & 87,2 & 87,0 \\
Cenizas & 8,4 & 9,3 \\
PB & 16,3 & 18,0 \\
GB & 1,0 & 1,2 \\
FND & 55,4 & 43,9 \\
FAD & 38,5 & 28,2 \\
LAD & 7,6 & 6,5 \\
CNF & 17,9 & 26,6 \\
Energía bruta, MJ/kg MS & 19,37 & 19,30 \\
\hline
\end{tabular}

${ }^{1}$ Composición (g/kg): $\mathrm{NaCl}, 970 ; \mathrm{Na}, 385 ; \mathrm{Mg}, 2 ; \mathrm{Zn}$, 0,81; Cu, 0,22; I, 0,1; Co, 0,018; Se, 0,01; vitamina A, $600000 \mathrm{UI}$; vitamina D3, $120000 \mathrm{UI}$; vitamina E, $1300 \mathrm{UI}$. que una de ellas tenía como fuente energética principal cebada (dieta C) y la otra maíz (dieta M). La fibra y la proteína la aportaba el heno de alfalfa y como concentrado proteico en ambas dietas se incorporó harina de girasol (pellets). También se incorporó en las dietas un bloque de sal-vitamínico-mineral (composición en tabla I), y agua limpia y fresca a voluntad.

La composición química de las raciones se analizó (por triplicado) siguiendo los métodos oficiales de análisis de AOAC (2000) para determinar materia seca (MS), cenizas, materia orgánica $(\mathrm{MO})$, proteína bruta (PB), grasa bruta (GB), fibra ácido detergente (FAD) y lignina ácido detergente (LAD). La fibra neutro detergente (FND), se determinó según Mertens (2002). La FND, FAD y LAD se determinaron utilizando la técnica de las bolsas de filtro de ANKOM. Para la determinación de los carbohidratos no fibrosos (CNF) se utilizó la ecuación de Miller y Hoover (1998). La concentración de energía de las dietas se determinó utilizando una bomba calorimétrica adiabática (Gallenkamp Autobomb. Loughborough, UK). En la tabla I se muestra específicamente la composición en ingredientes y composición química analizada de las dos dietas experimentales.

\section{Procedimiento experimental}

La temperatura y humedad relativa del aire estuvieron controladas durante toda la fase experimental mediante un sensor (HOBBO; software BoxCarPro3). La luz se mantuvo encendida de 07:00 a 19:00 horas.

Las cabras se alojaron en 3 jaulas de digestibilidad individuales provistas de un dispositivo para la recogida y separación de heces y de orina. El comedero estaba localizado en la parte exterior de la jaula, de modo que ninguna porción de comida podía ir a parar junto al excremento, y también era desmontable para el fácil manejo. El bebedero estaba dentro de la jaula y era automático. Inmediatamente debajo del suelo de la jaula, constituido por un enrejado metálico, se 


\section{LÓPEZ, RÓDENAS, PIQUER, CERISUELO, CERVERA Y FERNÁNDEZ}

sitúa una fina rejilla que retiene las heces y permite el paso de la orina, la cual resbala por una plancha metálica en forma de V, ligeramente inclinada hacia detrás, y desagua en un recipiente donde queda recogida. El recipiente contenía $50 \mathrm{cc}$ de una solución de ácido sulfúrico al $10 \%$, con objeto de evitar pérdidas de nitrógeno amoniacal.

Las cabras se pesaron al principio y al final del periodo experimental. El experimento consistió en una fase de adaptación a las jaulas y dietas de 7 días, seguido de una fase experimental de 5 días durante la cual se controló el consumo, producción de heces y orina. Este mismo procedimiento se repitió dos veces, una primera para la dieta $\mathrm{C}$ y una segunda para la dieta M. Ambos procedimientos se realizaron con los mismos animales durante el mes de Julio de 2009. La ración se ofrecía en dos tomas diarias, siempre a las mismas horas, 8:30 y 15:00 h.

Durante el periodo experimental se controló la ingestión de alimento diariamente mediante la recogida y peso diario del rehusado del alimento que se había ofrecido el día anterior. Las heces y la orina también se recogieron y pesaron todos los días, antes del suministro de la ración de la mañana.

Las heces de cada día eran congeladas a $-20^{\circ} \mathrm{C}$ hasta el momento de su análisis, en el que se homogeneizaron todas las muestras tomadas de cada animal y se analizó el homogeneizado de cada animal de toda la fase experimental.

La composición química de las heces se analizó (por triplicado) siguiendo los métodos oficiales de análisis de AOAC (2000) para determinar MS, cenizas, MO, PB, FAD y LAD y, según Mertens (2002) para determinar FND. La concentración energética en las heces se determinó utilizando una bomba calorimétrica (Gallenkamp Autobomb; Loughborough, UK). A partir de estos datos se calculó la digestibilidad de las dietas.

\section{MedidAs RESPIROMÉTRICAS}

Se utilizó una máscara de circuito abierto adaptada a pequeños rumiantes para medir la producción de calor de las cabras en mantenimiento mediante calorimetría indi-

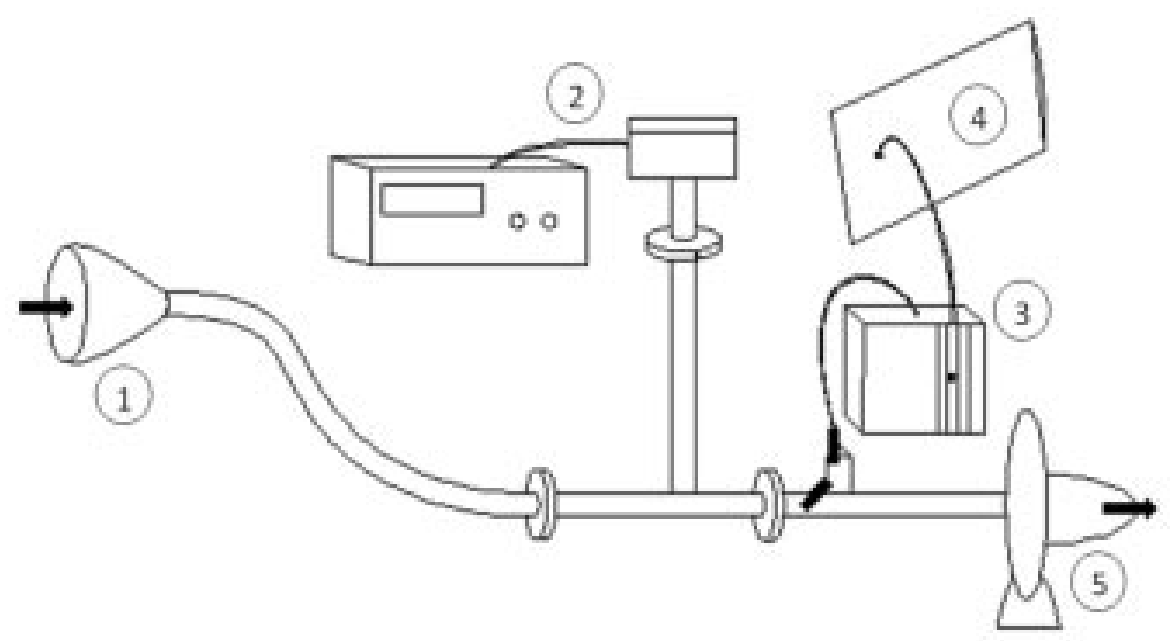

1: Máscara; 2: Caudalímetro y totalizador; 3: Bomba y caudalímetro; 4: Bolsa de recogida del aire; 5: Ventilador centrífugo.

Figura 1. Esquema de la máscara de circuito abierto para ganado caprino. (Diagram of open circuit respiration mask for goats). 


\section{DETERMINACIÓN DE PRODUCCIÓN DE METANO EN GANADO CAPRINO}

recta, y además se determinó de forma directa la producción de metano $\left(\mathrm{CH}_{4}\right)$ de los animales.

El cuarto día del periodo experimental, antes de ofrecer la primera toma diaria de alimento, se tomó una muestra del aire expirado de cada cabra, realizándose previamente un blanco con aire atmosférico. A continuación, se volcó el alimento en los comederos y las cabras comenzaron a comer. Una hora después de repartir la comida se retomaron las medidas de aire expirado por los animales. A partir de este momento, cada 35 minutos se repitió la toma de muestra de aire expirado a cada cabra, haciendo 3 mediciones por cabra a lo largo de la mañana. Entre las sucesivas medidas las cabras tenían a su disposición el alimento.

Cada medición consistió en recoger el aire expirado por el animal durante $10 \mathrm{minu}$ tos seguidos mediante una máscara de circuito abierto que se colocaba en la cabeza del animal. Este aire quedaba contenido en una bolsa-balón Douglas (PanLab S.L. Harvard Apparatus. Barcelona, España) con una capacidad de 15 litros, siendo el caudal de entrada a la bolsa de 90 1/hora. Para poder pasar una muestra del aire expirado a la bolsa era necesario conectar la máscara, mediante una tubería, a un caudalímetro (Sensyflow VT-S, ABB S.A. Zurich, Suiza) regulado para trabajar a un ritmo medio de 2000 litros de aire por hora. A su vez el caudalímetro estaba conectado a un ventilador centrífugo (CST60, Soler Palau S.A., Barcelona, España). El dispositivo completo fue diseñado pensando en minimizar las posibles pérdidas de carga por lo que se evitó cambios en el diámetro de las tuberías (figura 1).

Tras los 10 minutos de recogida de aire expirado, la bolsa se cerraba herméticamente y se llevaba al laboratorio, donde se conectaba a un analizador de gases (Easyflow 3020, ABB S.A. Zurich, Suiza) con cubetas de calibración incorporadas para medir concentraciones de oxígeno, dióxido de carbono y metano.

\section{ANÁLISISDE DATOS}

Los datos se analizaron mediante un análisis de varianza con el tipo de dieta como efecto fijo, los valores medios se compararon con un test de Duncan. Para ello se utilizó el software SPSS (2008) v16.

\section{RESULTADOSYDISCUSIÓN}

Las condiciones de temperatura ambiente y humedad relativa, que fueron controladas continuamente durante todo el experimento, estuvieron comprendidas entre 24 y $31^{\circ} \mathrm{C}$ y entre 62 y $77 \%$ respectivamente, valores habituales del mes de Julio en el cual se llevó a cabo el trabajo.

Los resultados obtenidos relativos a la digestibilidad de las dietas se muestran en la tabla II. No se encontraron diferencias significativas entre las dietas para la digestibilidad de las fracciones analizadas. Los animales de nuestro estudio fueron homogéneos en cuanto a edad, peso vivo y nivel productivo (mantenimiento) y, además las dietas eran isoenergéticas, cambiando sólo la fuente de cereal, como se observa en la tabla I. Como puede observarse en la tabla II, los coeficientes de digestibilidad para la MS y la EB fueron 69,2 y 68,6\%, respectivamente, similares a los que encuentran otros autores como Prieto et al. (1990) con machos castrados de raza Granadina en mante-

Tabla II. Coeficientes de digestibilidad de las dietas experimentales (\%). (Digestibility coefficients of experimental diets (\%)).

\begin{tabular}{lcccc}
\hline & Cebada & Maíz & ES & $\mathrm{p}$ \\
\hline MS & 68,3 & 70,1 & 1,34 & $\mathrm{~ns}$ \\
MO & 69,2 & 71,1 & 1,27 & $\mathrm{~ns}$ \\
PB & 62,0 & 63,4 & 2,41 & $\mathrm{~ns}$ \\
FND & 44,5 & 42,4 & 2,80 & $\mathrm{~ns}$ \\
Energía & 67,7 & 69,6 & 1,40 & $\mathrm{~ns}$ \\
\hline
\end{tabular}

ES: error estándar; ns: $p>0,05$. 


\section{LÓPEZ, RÓDENAS, PIQUER, CERISUELO, CERVERA Y FERNÁNDEZ}

nimiento, con dietas basadas en alfalfa peletizada y cebada $(63,9$ y $62,2 \%$, respectivamente). Bhatta et al. (2008) encontró valores del 65,0 y $66,8 \%$ respectivamente con caprino y 19 dietas mixtas.

Con aproximadamente un mismo peso vivo medio $(33,08 \pm 2,1)$, las cabras del grupo M consumieron $86 \mathrm{~g}$ más de MS por día que las del grupo $\mathrm{C}$, aunque las diferencias no fueron significativas. Como las dietas tenían el mismo contenido energético (19,33 MJ/kg de MS de media), y debido al ligero mayor consumo de MS de las cabras del grupo M, el consumo de EB también fue superior, aunque nuevamente no significativo (tabla III).

En cuanto a la influencia del tipo de cereal sobre la producción de $\mathrm{CH}_{4}$, puede verse en la tabla III que, antes de comer, la liberación de $\mathrm{CH}_{4}$ en el aire exhalado por las cabras (determinado por calorimetría indirecta mediante una máscara de circuito abierto) no presenta diferencias entre las dos dietas (con un valor medio de $0,201 \mathrm{CH}_{4} / \mathrm{h}$ ). Sin embargo, cuando se empezaron a realizar determinaciones sucesivas, tras una hora de espera después de ofrecer el alimento, se observó un aumento de la producción de $\mathrm{CH}_{4}$, siendo superior $(\mathrm{p}<0,05)$ cuando las

Tabla III. Efecto del grano de cereal sobre la producción de metano. (Cereal grain effect on methane production).

\begin{tabular}{lcccc}
\hline & Cebada & Maíz & ES & $\mathrm{p}$ \\
\hline $\mathrm{PV}, \mathrm{kg}$ & 33,50 & 32,67 & 2,10 & $\mathrm{~ns}$ \\
ICd, kg MS & 0,715 & 0,801 & 0,05 & $\mathrm{~ns}$ \\
EBD, MJ/kg MS & 19,37 & 19,30 & - & - \\
IECd, MJ & 13,77 & 15,45 & 0,91 & $\mathrm{~ns}$ \\
$\mathrm{PMAC}, / \mathrm{h}$ & 0,19 & 0,22 & 0,32 & $\mathrm{~ns}$ \\
$\mathrm{PMDC}, / \mathrm{h}$ & 0,69 & 0,77 & 0,04 & * \\
\hline
\end{tabular}

ES: error estándar; ns: $p>0,05 ;{ }^{*} p<0,05$. PV: peso vivo; ICd: Ingestión/cabra y día; EBD: energía bruta dietas; IECd: Ingestión de energía/ cabra/día; PMAC: Producción $\mathrm{CH}_{4}$ antes de comer; PMDC: Producción $\mathrm{CH}_{4}$ durante la comida. cabras comen la dieta que contiene maíz frente a la dieta con cebada $(0,69$ vs $0,77 \mathrm{l} / \mathrm{h}$ para la dieta con cebada y maíz respectivamente, media de 3 determinaciones).

Sobre el efecto del grano de cereal en la fermentación ruminal, el grano de cebada es más fermentable que el grano de maíz, este último con un menor contenido de endospermo harinoso y potencialmente menos degradable. Según la literatura, la suplementación con fuentes de almidón menos degradables es una vía para reducir las pérdidas de metano (Crutzen et al., 1986; Johnson and Johnson, 1995; Moss et al., 2000; Benchaar et al., 2001; Beauchemin y McGinn, 2005), aunque estos autores no evalúan la interacción del almidón con otros concentrados de la ración. Así por ejemplo en el trabajo de Beauchemin y McGinn (2005) se muestra que la sustitución de grano de cebada por grano de maíz reduce las emisiones de metano desde un 4,03 hasta un $2,81 \%$ de la energía bruta ingerida en raciones concentradas (dicha reducción se observa en la fase de acabado del cebo de terneros y con una ración basada en ensilado de cebada y cebada o maíz grano).

Los resultados obtenidos muestran una tendencia contraria, probablemente esta tendencia sea debida al nivel de alimentación que era próximo al mantenimiento y, a la sincronización en la degradación de la fuente energética (cebada) y proteica (harina de girasol), es decir, las velocidades de degradación de la cebada y de la harina de girasol son similares, quedando menos hidrógeno libre en el rumen para la formación de metano (Russell y Gahr, 2000). En la dieta con maíz el almidón se degrada más lentamente, por lo tanto no hay sincronía con la fuente proteica (harina de girasol) y puede dar lugar a más partículas groseras de almidón sin degradar en el rumen que favorecen el desarrollo de protozoos y, por lo tanto, la proliferación de microorganismos metanogénicos a nivel ruminal; una mayor producción de hidrógeno que los microorganismos 


\section{DETERMINACIÓN DE PRODUCCIÓN DE METANO EN GANADO CAPRINO}

Tabla IV. Producción de $\mathrm{CH}_{4}$ y valores de $\mathrm{Ym}$. ( $\mathrm{CH}_{4}$ production and $\mathrm{Ym}$ values).

\begin{tabular}{lcccc}
\hline & Cebada & Maíz & ES & $\mathrm{p}$ \\
\hline Producción media $\mathrm{CH}_{4}, \mathrm{l} / \mathrm{h}$ & 0,44 & 0,50 & 0,01 & - \\
$\mathrm{CH}_{4}$ media, $\mathrm{kJ} / \mathrm{cabra}$ y día & 417,54 & 474,48 & 12,7 & - \\
$\mathrm{Ym}$ medio & 3,11 & 3,09 & 0,20 & $\mathrm{~ns}$ \\
$\mathrm{CH}_{4}$ durante comida, kJ/cabra y día & 654,78 & 730,70 & 16,97 & $*$ \\
Ym, durante comida & 4,88 & 4,76 & 0,32 & $\mathrm{~ns}$ \\
Valores estimados según diferentes autores & & & & \\
Ym (Aguilera y Prieto, 1991) [1] & 7,64 & 7,92 & \\
Ym (de Blas et al., 2008) [2] & 5,87 & 5,69 & & \\
\hline
\end{tabular}

Ym: fracción de la EB del alimento que se transforma en $\mathrm{CH}_{4}$.

ES: error estándar; ns: $p>0,05 ;{ }^{*} p<0,05$.

metanogénicos utilizan para reducir el carbono del $\mathrm{CO}_{2} \mathrm{a} \mathrm{CH}_{4}$ (Hindrichsen et al., 2006).

A nivel práctico, las pérdidas de $\mathrm{CH}_{4}$ se expresan normalmente como fracción de la EB ingerida, y a este término se le conoce como Ym. Dichos valores son esenciales para la elaboración del inventario de emisiones de gases que tiene que realizar cada país (IPCC, 2006), y hay una importante escasez de datos reales. Cuando expresamos la producción de $\mathrm{CH}_{4}$ en función de la EB ingerida no se observaron diferencias significativas entre las dietas. En la tabla IV se muestra la producción media obtenida de metano (antes y durante la comida), y se calcula el factor de conversión de $\mathrm{CH}_{4}(\mathrm{Ym})$ a partir de nuestros resultados y a partir de ecuaciones representativas de la bibliografía. En el caso del Ym medio (medidas antes y después de comer) no se encuentra efecto de la dieta (valores medios de 3,1 y 4,8 respectivamente).

Como se indica en las guías del IPCC (IPCC, 1996; IPCC, 2006), el valor de Ym depende de la calidad y digestibilidad de las distintas dietas. Dada la relación negativa que existe entre el Ym y la digestibilidad, se asume que a medida que aumenta la digestibilidad disminuye el Ym. Las diferencias numéricas que encontramos son debidas a que Ym calculada se hace a partir de la EB ingerida, $\mathrm{y}$ las estimadas por las ecuaciones de la bibliografía se hacen a partir del coeficiente de digestibilidad de la energía (que en nuestro caso fue numéricamente superior para el maíz).

Los valores de Ym que se encuentran en la bibliografía varían entre el $2 \%$ y el $11 \%$ de la energía ingerida para distintas dietas (Johnson y Johnson, 1995). Los valores obtenidos en este trabajo se encuentran dentro de este intervalo, más bien hacia el límite inferior debido a que nuestras raciones no están basadas en pastos.

Aguilera y Prieto (1991) establecieron la siguiente ecuación de regresión lineal para ganado caprino

$$
Y m=0,151 \times d E-2,58
$$

donde:

$\mathrm{Ym}=$ Factor de conversión de $\mathrm{CH}_{4}$ (fracción de la EB del alimento que se transforma en $\mathrm{CH}_{4}$ ); $\mathrm{dE}=$ Digestibilidad aparente de la energía $(\%)$.

Estos autores encontraron una correlación positiva entre la producción de metano y la digestibilidad energética de la dieta, y los valores de Ym estimados con esta ecuación son superiores a los observados. Estos autores trabajaron desde la perspectiva del ganado caprino como un animal para pastorear y ramonear. La situación que nos encontramos en España y en muchos 


\section{LÓPEZ, RÓDENAS, PIQUER, CERISUELO, CERVERA Y FERNÁNDEZ}

países europeos es el de la utilización de dietas mixtas o concentradas y ganado estabulado.

En estas circunstancias de Blas et al. (2008) observaron una correlación negativa entre la concentración energética de la dieta y la producción de metano. Seguidamente se presenta la ecuación obtenida como resultado de una amplia revisión de ensayos recientes

$$
Y m=-0,096 \times d E+12,367
$$

donde:

$\mathrm{Ym}=$ Factor de conversión de $\mathrm{CH}_{4}$ (fracción de la

EB del alimento que se transforma en $\mathrm{CH}_{4}$ ); $\mathrm{dE}=$ Digestibilidad aparente de la energía (\%).

Si utilizamos la ecuación de de Blas et al. (2008), las dietas experimentales estudiadas se encuentran con un Ym en torno a un 5,7 (valor más próximo al obtenido a partir del ensayo; $\mathrm{Ym}=4,8)$. De Blas et al. (2008)

\section{BIBLIOGRAFÍA}

Aguilera, J.F. and Prieto, C. 1991. Methane production in goats given diets based on lucerne hay and barley. Arch. Anim. Nutr., 41: 77-84.

AOAC. 2000. Official methods of analysis of the AOAC International. $17^{\text {th }}$ ed. Association of Official Analytical Chemist. Arlington. USA.

Beauchemin, K.A. and McGinn, S.M. 2005. Methane emissions from feedlot cattle fed barley or corn diets. J. Anim. Sci., 83: 653-661.

Benchaar, C., Pomar, C. and Chiquette, J. 2001. Evaluation of dietary strategies to reduce methane production in ruminants: A modeling approach. Can. J. Anim. Sci., 81: 563-574.

Bhatta, R., Enishi, O., Takusari, N., Higuchi, K., Nonaka, I. and Kurihara, M. 2008. Diet effects on methane production by goats and a comparison between measurement methodologies. J. Agric. Sci., 146: 705-715.

CAPRA v.1. 2008. Racionamiento para ganado caprino lechero. Ed. Universidad Politécnica de Valencia. Valencia. $2165 \mathrm{pp}$.

Crutzen, P.J., Aselman, I. and Seiler, W. 1986. Methane production by domestic animals, wild observaron que los valores medios de Ym se reducen desde alrededor de un $6,5-7,5 \%$ en raciones extensivas hasta un $4,0-4,5 \%$ en raciones concentradas. El factor de conversión medio de $\mathrm{CH}_{4}$ medio para las dietas mixtas estudiadas en este estudio fue de 4,8 , inferior al estimado por de Blas et al. (2008) aunque hay que destacar que las cabras estaban en mantenimiento.

\section{CONCLUSIONES}

Es posible cuantificar la producción de $\mathrm{CH}_{4}$ por respirometría y detectar variaciones entre dietas. El factor de conversión de $\mathrm{CH}_{4}$ en ganado caprino en mantenimiento alimentado con dietas mixtas fue de 4,8 . Aunque dicha información es esencial para elaborar los inventarios de emisiones de gases por la ganadería, son necesarios más estudios en donde podamos evaluar factores ligados al animal, a los alimentos y a las condiciones medioambientales.

ruminants, other herbivorous fauna, and humans. Tellus, 38 (B): 271-284.

De Blas, C., García-Rebollar, P., Cambra-López, M. y Torres, A.G. 2008. Contribución de los rumiantes a las emisiones de gases con efecto invernadero. XXIV Curso de especialización FEDNA. Editorial FEDNA. Madrid. pp. 121-150. Hindrichsen, I.K., Wettstein, H.R., Machmuller, A. and Kreuzer, M. 2006. Methane emission, nutrient degradation and nitrogen turnover in dairy cows and their slurry at different milk production scenarios with and without concentrate supplementation. Agr. Ecosyst. Environ., 113: 150-161.

INRA. 2007. Alimentation des bovins, ovins et caprins. Besoins des animaux. Valeurs des aliments. Ed. Quae. Paris. 307 pp.

IPPC. 1996. Revised 1996 IPPC Guidelines for national greenhouse gas inventories. Chapter 4. Agriculture. IPPC/OECD/IEA. Paris. $140 \mathrm{pp}$.

IPCC. 2001. Climate change 2001: The scientific basis. In: J.T. Houghton (Ed.). Intergovernamental Panel on Climate Change (IPCC). Cambridge 


\section{DETERMINACIÓN DE PRODUCCIÓN DE METANO EN GANADO CAPRINO}

University Press. Ginebra. 944 pp.

IPCC. 2006. 2006 IPCC Guidelines for national greenhouse gas inventories. Vol. 4. Agriculture, forestry and land use. IGES. Kanagawa. Japón. Chapter 10. pp. 10.1-10.87.

Johnson, K.A. and Johnson, D.E. 1995. Methane emissions in cattle. J. Anim. Sci., 73: 24832492.

Khalil, M.A.K. 2000. Atmospheric methane: An introduction. In: M.A.K. Khalil (Ed.). Atmospheric methane, its role in the global environment. Springer-Verlag. Berlín. pp. 1-8.

Kreuzer, M. and Hindrichsen, I.K. 2006. Methane mitigation in ruminants by dietary means: The role of their methane emission from manure. International Congress Series. In: C.R. Soliva, J. Takahashi and M. Kreuzer (Eds.). Greenhouse gases and animal agriculture: An update. Elsevier. The Netherlands. International Congress Series, 1293: 199-208.

Lachica, M. and Aguilera, J.F. 2003. Estimation of energy needs in the free-ranging goat with particular reference to the assessment of its energy expenditure by the 13C-bicarbonate method. Small Rumin. Res., 49: 303-318.

Lana, R.P., Russell, J.B. and Van Amburgh, M.E. 1998. The role of $\mathrm{pH}$ in regulating ruminal methane and ammonia production. J. Anim. Sci., 76: 2190-2196.

Mertens, D.R. 2002. Gravimetric determination of amylase-treated neutral detergent fibre in feeds with refluxing beakers or crucibles: collaborative study. J. AOAC Int., 85: 1217-1240.

Miller, T.K. and Hoover, W.H. 1998. Nutrient analyses of feedstuff including carbohydrates. West Va. Univ. Morgantown, WV. Anim. Sci. Report, ํㅡ 1 .

Ministerio de Medio Ambiente. 2007. Inventario de emisiones de gases de efecto invernadero de España. Años 1900-2005. Comunicación a la Comisión Europea. Decisiones 280/2004/CE y 2005/166/CE. Ministerio de Medio Ambiente. Madrid. España. $330 \mathrm{pp}$.

Moss, A.R., Jouany, J.P. and Newbold, J. 2000. Methane production by ruminants: its contribution to global warning. Ann. Zootech., 49:231-253.

O'Kelly, J.C. and Spiers, W.G. 1992. Effect of monensin on methane and heat productions of steers fed lucerne hay. Aust. J. Agric. Res., 43: 1789-1793.

Prieto, C., Aguilera, J.F., Lara, L. and Fonollá, J. 1990. Protein and energy requeriments for maintenance of indigenous Granadina goats. Brit. J. Nutr., 63: 155-163.

Russel, R.W. and Gahr, S.A. 2000. Glucose availability and associated metabolism. In: J.P.F. D'Mello (Ed.). Farm Animal Metabolism and Nutrition. CAB Intl. Publ. Wallingford. Oxon. UK. pp. 121-147.

SPSS. 2008. Statistical Package for the Social Sciences. Versión 16.0 para Windows. SPSS Inc. Chicago. II. EEUU.

Van Soest, P.J. 1994. Nutritional ecology of the ruminant. $2^{\mathrm{a}} \mathrm{Ed}$. Cornell University Press. Comstock. 476 pp.

Wuebbles, D.J. and Hayhoe, K. 2002. Atmospheric methane and global change. Earth-Sci. Rev., 57: 177-210. 